\title{
Creating a soluble binder to endothelin-1 based on the natural ligand binding domains of the endothelin-1 (G-protein-coupled) receptor
}

\author{
Arjun Jain ${ }^{1,2}$, Vidhi Mehrotra ${ }^{1}$, Hannah Yong ${ }^{2}$, Kirti Hiremath ${ }^{3}$, Ashok Jain ${ }^{1}$, Martin Johnson ${ }^{2}$, Ira Jha ${ }^{4,5}$ \\ ${ }^{1}$ Accelerate Cambridge, Judge Business School, University of Cambridge, Cambridge, United Kingdom \\ ${ }^{2}$ Department of Physiology, Development and Neuroscience, University of Cambridge, Cambridge, United \\ Kingdom \\ ${ }^{3}$ University of Delhi, Delhi, India \\ ${ }^{4}$ National University of Singapore, Singapore \\ ${ }^{5}$ Indian Institute of Management, Ahmedabad, India \\ Correspondence: Dr Arjun Jain, e-mail: jain_arjun@yahoo.com, phone: +4401223660488
}

Running title: Creating a soluble form of the ET-1 receptor

\begin{abstract}
Since its discovery in 1989, there has been extensive research on endothelin (ET)-1 physiology, as well as pathology. Accordingly, there is considerable research on the discovery of therapeutics based around ET-1, amongst which current treatment options include endothelin receptor antagonists. These target the ET-1 receptors, which are Gprotein-coupled receptors (GPCRs). We have effectively developed a soluble form of a GPCR that binds to ligands, by constructing a fusion polypeptide of different endothelin receptor ligand binding domains. Phage experiments identified strong binders to ET-1. We then constructed Fc-fusions of the top binders and further binding assays revealed $a K_{D}$ of $21.2 \mathrm{nM}$ for the Fc-ETtr1 construct and $\mathrm{K}_{\mathrm{D}}$ of $77.3 \mathrm{nM}$ for the FC- ETtr2 construct. These constructs are soluble and have the ability to bind and potentially sequester elevated ET-1 levels that are prevalent in different diseases. These results provide a novel approach to targeting GPCR-binding ligands, and thereby to contribute to a very important class of therapeutics.
\end{abstract}




\section{Abbreviations}

$\begin{array}{ll}\text { ECL } & \text { extracellular loop } \\ \text { ET-1 } & \text { endothelin-1 } \\ \text { ETA } & \text { endothelin A } \\ \text { ETB } & \text { endothelin B } \\ \text { ETtr } & \text { endothelin-1 traps } \\ \text { FFP } & \text { Fc-fusion protein } \\ \text { GPCR } & \text { G-protein -coupled receptor } \\ \text { HA epitope } & \text { human influenza hemagglutinin epitope } \\ \text { LBD } & \text { ligand binding domain } \\ \text { PBST } & \text { phosphate buffered saline tween } \\ \text { SB } & \text { super broth medium } \\ \text { STR } & \text { streptavidin }\end{array}$

\section{Introduction}

Endothelins are a family of vasoactive peptides that have key physiological functions in normal tissue, acting as modulators of the vascular tone, tissue differentiation, cell proliferation, development and hormone production [1]. The family of endothelins comprises three isoforms each of 21 amino acids (ET-1, ET-2, ET-3). ET-1 is the most abundant member of the family of endothelins [2].

ET-1 exerts its effects by binding to the endothelin A (ETA) and endothelin B (ETB) receptors, two highly homologous cell-surface proteins that belong to the G-protein-coupled receptor superfamily [3]. The two receptors share about $60 \%$ similarity in the primary structure [1]. The ETA receptor shows different affinities to the three endothelin isoforms in the order ET$1 \geq$ ET-2 > > ET-3 [4], while the ETB receptor binds to ET-1, ET-2 and ET-3 with an almost equal affinity [5].

Previous studies have described different ET receptor antagonists, which compete with endothelins for binding to its receptors and block G-protein-mediated signal transduction [4]. The ET receptor antagonists include ETA receptor-specific and ETB receptor-specific 
antagonists, as well as dual receptor antagonists, such as Bosentan. Needless to say, given the relevance of ET-1 to different disease pathologies, these antagonists have important therapeutic value. Indeed, some of these are already in clinical use $[6,7]$.

In addition, studies using ET antagonists have helped delineate the mechanism of ET-1 (ligand) binding to its receptors. For example, BQ123 is a novel cyclic pentapeptide, cyclo(D-Trp-D-Asp-L-Pro-D-Val-L-Leu-), which binds to the ET-1-selective ETA receptor but not to the ETB receptor that binds ligands non-selectively [8]. Studies with this antagonist have helped identify the B-loop domain of the ETA receptor as important for ligand binding [4], a conclusion based on the observation that grafting the B-loop of the ETA receptor onto the corresponding domain of the ETB receptor renders the resulting chimeric receptor sensitive to antagonism by BQ123.

Previous work has also found that a part of the ETA receptor $\mathrm{N}$-terminal domain in close proximity to the first transmembrane region and a 5 amino acid sequence (140-KLLAG-144) are important elements for ligand binding [4]. Furthermore, the C-terminal 8-amino acid residues located in close proximity to the seventh transmembrane region and the C-terminal 16-amino acid residues in the third intracellular loop are important for the binding of ET-1. These seem to contribute to the three-dimensional structure of the ligand-binding site located in the extracellular domains.

Based on our understanding of ET-1 binding to its receptors, we have constructed fusion polypeptides encompassing the different ETA receptor ligand binding domains (LBDs). We have chosen to proceed with the ETA receptor LBDs, because they exhibit the highest specificity to ET-1 over its isoforms. Phage-display experiments (Figure 1) have been used to investigate the binding potential of these different fusion polypeptides, as described in this paper. Phage display is one of the most powerful and widely used laboratory techniques for the study of protein-protein, protein-peptide and protein-DNA interactions. This technology is mainly based on displaying the protein or peptide of interest, which in this case is endothelin-1.

\#Figure 1 


\section{Methods}

\section{Cloning and phage production}

ETA receptor LBD constructs were ordered as synthetic DNA (Genscript, Piscataway, NJ, USA) and cloned with Sfil-sites into a phagemid vector pHB32x, which is a modified vector of pEB32x containing a loxP site after the truncated p3-gene [9]. The constructs were verified by sequencing and transformed into E. coli strain SS320 for phage production. The cells were grown in $5 \mathrm{ml}$ super broth medium (SB) containing $25 \mu \mathrm{g} / \mathrm{ml}$ chloramphenicol and $10 \mu \mathrm{g} / \mathrm{ml}$ tetracycline at $37{ }^{\circ} \mathrm{C}$ with $250 \mathrm{rpm}$ shaking to mid-log phase and infected with helper phage VCSM13 for $1 \mathrm{~h}$ at $37{ }^{\circ} \mathrm{C}$. Kanamycin was added to a final $50 \mu \mathrm{g} / \mathrm{ml}$ concentration and the cultures were grown over night at $30^{\circ} \mathrm{C}$ with $250 \mathrm{rpm}$ shaking. Phage stocks were prepared with two sequential PEG/NaCl-precipitations according to a standard protocol to a final volume of $500 \mu \mathrm{l}$ PBS. Phage titers were determined by quantitative PCR, targeting the truncated p3-gene using previously titered phage stock pHB32x-SpyC as a standard.

The 5 different constructs ordered from Genscript are designated ETtr1-5. These constructs vary in the combination of LBDs that constitute each fusion polypeptide (see Figure 3 for details of LBDs in each construct); the fusions are connected by an EAAAK amino acid sequence.

\section{Phage immunoassay}

Anti-HA phage immunoassays were performed by diluting $5 \times 10^{9} \mathrm{cfu}$ phage from each display stock in $500 \mu$ phosphate buffered saline tween (PBST 0.05) $+1 \% \mathrm{w} / \mathrm{v}$ bovine serum albumin (BSA). All incubations were performed at room temperature. The phage samples were mixed with $100 \mu \mathrm{g}$ of prewashed paramagnetic Dynabeads MyOne Streptavidin C1 (Invitrogen) to analyse nonspecific background binding. The phage samples were also mixed with $100 \mu \mathrm{g}$ Pierce Anti-HA Magnetic Beads (Thermo Scientific) for displayed HA-epitope detection. ET-1 phage immunoassays were performed by diluting $2.5 \times 10^{10} \mathrm{cfu}$ phage from each display stock in $500 \mu \mathrm{l}$ PBST0.05 $+1 \% \mathrm{w} / \mathrm{v}$ BSA and mixed with $100 \mu \mathrm{g}$ MyOne beads pre-coated with biotinylated ET-1 (Phoenix Pharmaceuticals). Biotinylated ET-1 was coated at $50 \mathrm{ng} / \mu \mathrm{l}$ concentration on $1800 \mu \mathrm{g}$ MyOne Dynabeads for $30 \mathrm{~min}$, mixed by rotation, and washed three times with $1 \mathrm{ml}$ PBSTO.1 before the binding assay. Phages were allowed to 
bind for $30 \mathrm{~min}$ and $60 \mathrm{~min}$ in the anti-HA and ET-1 binding assays, respectively, and washed twice with $1 \mathrm{ml}$ PBST0.1. The bound phages were labelled with $100 \mu \mathrm{l}$ 1/1000 diluted horseradish peroxidase (HRP)/anti-M13 monoclonal conjugate in PBST0.05 + 3\% w/v fatfree milk powder for 30 min whilst being rotated. Beads were washed three times with PBST0.1 and transferred to PCR strips. Bead pellets were resuspended in $100 \mu \mathrm{l}$ TMB substrate, vortexed and incubated for $3 \mathrm{~min}$ to let the colour develop. Beads were collected on a magnet and supernatants transferred into a transparent 96-well plate containing $100 \mu \mathrm{l}$ $1 \mathrm{~N} \mathrm{H}_{2} \mathrm{SO}_{4}$ to stop the reaction. The absorbance was measured at $450 \mathrm{~nm}$ with SpectraMax plate reader.

Note: Empty MyOne/anti-HA beads and unrelated (HA-) pHB32x-SpyCatcher phage stock were included as negative controls.

\section{Creating an Fc-fusion construct and measuring binding affinity to ET-1}

The gene for the fusion protein was designed and optimised for expression in mammalian cells (HEK293) prior to being synthesised. The sequence was then subcloned into a cloning and expression vector for human Fc fusion proteins.

The Fc constructs were generated using the services of Absolute Antibody Ltd (Oxford, UK). In brief, HEK293 cells were passaged to the optimum stage for transient transfection. Cells were transiently transfected with the appropriate expression vector and cultured for a further 6-14 days. An appropriate volume of cells was transfected with the aim of obtaining 1-5 mg of purified Fc fusion protein. Cultures were harvested and a one-step purification performed using affinity chromatography.

For this, culture supernatant containing Fc fusion protein was loaded onto a MabSelect SuRe Protein A column at $4 \mathrm{ml} / \mathrm{min}$ and washed with phosphate buffered saline (PBS) pH 7.2. A step elution was performed with sodium citrate $\mathrm{pH} 3.0$ buffer. Eluted protein was neutralised with $10 \%(\mathrm{v} / \mathrm{v})$ Tris $\mathrm{pH}$ 9.0. Upon successful purification, the Fc fusion protein was buffer exchanged into PBS pH 7.4. The protein was analysed for purity by SDS-PAGE and concentration determined by UV spectroscopy (at $280 \mathrm{~nm}$ ). 
For the binding affinity measurement, we employed the use of the Octet Red96 system [10]. For this, we fixed biotinylated ET-1 (Phoenix Pharmaceuticals) and then probed with our Fcfusion construct.

\section{Binding affinity measurement}

The kinetics of FC-ETtr1 and Fc-ETtr2 binding to biotinylated-endothelin-1 was determined using the Octet Red96 system (ForteBio, Menlo Park, CA). The buffer for the assays was phosphate buffered saline (PBS) with $0.01 \%(\mathrm{w} / \mathrm{v})$ bovine serum albumin (BSA) and $0.002 \%$ Tween-20. The measurements were carried out at $30{ }^{\circ} \mathrm{C} .1 \mu \mathrm{g} / \mathrm{ml}$ bio-endothelin-1 was captured on dip-and-read STR (streptavidin) sensors, followed by binding of Fc-ETtr1 and FcETtr2 at 500 nM concentration. The ForteBio Octet analysis software (ForteBio, Menlo Park, CA) was used to generate the sensorgram. The signal from an endothelin-mounted tip without the Fc-fusion was used as a reference and subtracted from the sample signals.

\section{Statistics}

Statistical analysis was performed by analysis of variance (Fisher's Protected Least Significant Difference test), with $p<0.001$ being considered significant. 


\section{Results}

For phage display, the endothelin binding constructs were cloned as a fusion to the truncated g3p (g3p-CT) of filamentous phage. HA epitope, YPYDVPDYA, was inserted in all display constructs between the protein of interest and the g3p-CT to assess the display performance. The HA-epitope was detected on all phage samples by binding $5 \times 10^{9} \mathrm{cfu}$ display phage particles on anti-HA coated beads indicating that the constructs were displayed on the phage coat (Figure 2).

\#Figure 2

The binding of the ETtr constructs to ET-1 was then analysed on streptavidin beads precoated to saturation with biotinylated ET-1; $n=3$ (Figure 3). As mentioned in the Introduction section, Phage display is a powerful technique commonly used today to identify different protein-protein interactions.

\#Figure 3

Of the different peptides tested, ETtr1 and ETtr2 exhibited statistically significant binding to biotinylated ET-1 as deduced from application of an analysis of variance (Fisher's Protected Least Significant Difference) test; $n=3, p=0.0007$.

As a next step, we proceeded to construct Fc fusion proteins of ETtr1 and ETtr2. SDS-PAGE analysis confirmed that both constructs were successfully expressed as identified by their respective molecular weights: 93.7kDa for ETtr1 and 88.9kDA for ETtr2 (Figure 4).

\#Figure 4

Both constructs were expressed and isolated with a purity of $>98 \%$. Using these constructs, we then proceeded to analyse the binding affinity of each soluble construct. The first set of binding experiments did not exhibit any binding to bioendothelin-1 with either Fc-ETtr1 or Fc-ETtr2 (Figure 5A and B).

\#Figure $5 \mathrm{~A}$ and $\mathrm{B}$

We did not observe any binding of either dimeric Fc-fusion construct (Fc-ETtr1 or Fc-ETtr2). This was despite the strong binding obtained in our phage experiments (Figure 3). It is likely that the unpaired cysteine residues in each arm of our construct cross-link to form 
disulphide bridges that conceal the ligand-binding site in such a way that we do not observe any ET-1 binding. Breaking these disulphide linkages with a mild treatment of DTT, did restore ET-1 binding of our constructs (Figure $6 \mathrm{~A}$ and $\mathrm{B}$ ).

\#Figure $6 \mathrm{~A}$ and $\mathrm{B}$

The binding assay revealed a strong binding of the monomeric Fc-ETtr1 construct; mean binding affinity of $21.2 \mathrm{nM}(\mathrm{n}=6$; see table 1). The binding affinity of the monomeric Fc-ETtr2 construct was found to be weaker at $77.3 \mathrm{nM}\left(\mathrm{n}=3 ; \mathrm{K}_{\mathrm{D}}\right.$ error $\left.=1.567 \mathrm{E}-09\right)$. It is important to note that any effects of DTT were controlled by use of just 'buffer + DTT', which gave no binding to ET-1. The signal recorded for each monomeric FC-ETtr construct is with subtraction of the corresponding control, i.e. 'buffer + DTT'.

Table 1. Binding data for the monomeric Fc-ETtr1 construct.

\begin{tabular}{|l|l|l|}
\hline Sample & $K_{\mathrm{D}}(\mathrm{M})$ & $\mathrm{K}_{\mathrm{D}}$ Error \\
\hline Fc-ETtr1 & $2.159 \mathrm{E}-08$ & $6.774 \mathrm{E}-10$ \\
\hline Fc-ETtr1 & $2.186 \mathrm{E}-08$ & $6.956 \mathrm{E}-10$ \\
\hline Fc-ETtr1 & $2.535 \mathrm{E}-08$ & $7.901 \mathrm{E}-10$ \\
\hline Fc-ETtr1 & $1.861 \mathrm{E}-08$ & $6.260 \mathrm{E}-10$ \\
\hline Fc-ETtr1 & $2.194 \mathrm{E}-08$ & $1.525 \mathrm{E}-09$ \\
\hline Fc-ETtr1 & $1.787 \mathrm{E}-08$ & $1.295 \mathrm{E}-09$ \\
\hline
\end{tabular}

The binding assay revealed a strong binding of the monomeric Fc-ETtr1 construct; mean binding affinity of $2.120 \times 10^{-8} M$, i.e. $21.2 \mathrm{nM}(\mathrm{n}=6)$. 


\section{Discussion}

In this study, we constructed fusion polypeptides encompassing the different ETA receptor ligand binding domains, which exhibited the highest specificity to ET-1 over its isoforms, i.e. ET-2 and ET-3. We connected the different ETA receptor LBDs using rigid linkers, which are alpha helix-forming linkers with the sequence of $(E A A A K)_{n}$. As has been previously suggested, many natural linkers exhibit $\alpha$-helical structures, which are rigid and stable, with intra-segment hydrogen bonds and a closely packed backbone $[11,12]$. Therefore, the stiff $\alpha$-helical linkers may act as rigid spacers between protein domains, which would allow the ETA receptor extracellular loops (ECLS) to retain their structure to a higher degree once dissociated from their natural configuration as a part of a GPCR.

We used 'EAAAK' linkers before and after the ECL domains, because the ET receptor extracellular loops needed to be between helices, as suggested in the previous study by Orry and Wallace (2000), which produced a model of the ETA receptor and ET-1 ligand binding this GPCR [13].

Our results show that constructs ETtr1 and ETtr2 give statistically significant binding to biotinylated ET-1. Constructs ETtr1 and ETtr2 contain ICL3 (16 amino acids) and the Cterminus ( 8 amino acids) domains, which are lacking in construct ETtr4 and ETtr5. Therefore, either one or both of these domains are important for ET-1 binding. Furthermore, ECL2 is lacking in ETtr3 (which gave only very weak binding to biotinylated ET-1) but is present in ETtr1 and ETtr2 (Figure 3). Hence, the ECL2 domain also appears to be important for ET-1 binding.

As a next step, we created Fc-fusion constructs of ETtr1 and ETtr2 (that gave statistically significant binding to biotinylated ET-1 in our phage experiments). The binding affinity of these constructs to ET-1 was then measured, as the Fc-fusion construct is what may eventually be used as a drug. Fc-fusion proteins form an important class of therapeutics as evinced by the successful application of cytokine traps for patients with rheumatoid arthritis and other autoimmune disorders $[14,15]$. Our study confirmed the solubility of our constructs and revealed a strong binding affinity of $21.2 \mathrm{nM}(\mathrm{n}=6)$ for the monomeric FcETtr1 construct. 
Interestingly, ETtr1 gave stronger binding than the ETtr2 construct. This highlights the importance of the third extracellular loop domain (D-loop), which is present in ETtr1 but absent from ETtr2. Previous research has shown that both the C- and D- loops of the ETA receptor are responsible for ligand (ET-1) selection [4].

Next steps in our project include the current development of a monomeric Fc-ETtr1 construct (in order to circumvent use of DTT to generate monomers from our dimeric FC construct), followed by characterisation of its binding affinity. This would confirm that crosslinking of cysteine residues in our current dimeric Fc construct is an issue for ligand binding. Alternative designs for creating Fc constructs are detailed in Figure 7. These formats would circumvent any issues of cross-linking of cysteine residues, as was observed earlier in our study.

\#Figure 7

Fcy recombinant forms a group of recombinant proteins called Fc-fusion proteins (FFPs). Our ET-traps are also an Fc-fusion protein. FFPs are widely used in drug discovery, drug delivery, vaccine design and experimental research on receptor-ligand interactions. These fusion proteins have become successful alternatives to monoclonal antibodies for drug developments [16]. In all, "the customizable functionality of FFPs creates advantages over antibody-based therapies by combining modular building blocks that can reach targets not accessible to antibodies. Additional advantages include lower patient dosing, reduced production costs, and improved product homogeneity" (PEGS Boston, April 2016). Therefore, our Fc-fusion ET-trap may provide a novel therapeutic tool, which remains to be tested in the next phase of this project.

In summary, we have developed a soluble binder to our target ligand (ET-1), which in physiology binds a GPCR. These constructs consist of different peptide sequences that have been identified as strong binders to our target ligand (based on previous research). To our knowledge, we are the first to have reported the development of a soluble form of a GPCR in terms of its ligand binding ability. Although our study was based on ET-1, the same strategy may be applied to other GPCR-ligand systems, which may lead to the discovery of novel therapeutics in different disease areas. 


\section{Importance + Future opportunities}

Thus far, we have used phage display experiments to identify strong binders to ET-1. We have also expressed the top-binders as Fc-fusion proteins, which confirmed their solubility and allowed us to determine their binding affinity. The next steps in our project will be to apply affinity maturation to our constructs to further improve its binding affinity for ET- 1 . We also intend to investigate the possibility of using the ETB receptor ligand binding domains for construction of ET-traps. Previous studies have found that the ETB receptor strongly binds ET-1 but without specificity over its isoforms as found with the ETA receptor [17]. Hence, we would aim to develop a combination fusion polypeptide that encompasses both the strong ligand binding ETB receptor domains, as well as the ET-1-ligand specific ETA receptor domains.

Our aim will be to test the efficacy of this construct as a therapeutic tool. We intend to investigate the therapeutic effects of ET-traps in pre-eclampsia disease. Extensive research has implicated the vasoactive peptide, ET-1, as a major pathological factor in pre-eclampsia [18-21]. Clinical data have confirmed that ET-1 levels are significantly elevated in the blood of women with pre-eclampsia. ET-1 levels have been found to be almost ten times higher at $35-50 \mathrm{pg} / \mathrm{ml}$ in women with pre-eclampsia compared with $5-10 \mathrm{pg} / \mathrm{ml}$ in normotensive controls [18]. Increased ET-1 has also been shown to be a primary event in the pathophysiology of pre-eclampsia, being a stimulus of inflammation, hypertension and coagulation, which are all key characteristics of the disease. Previous work has shown that different markers of pathology are reduced significantly upon sequestering ET-1 [21]. If successful to reach the market, ET-traps would offer a long overdue treatment option for pregnancy disorders, such as pre-eclampsia.

Elevated ET-1 levels are also implicated in a host of other diseases as well, including cardiovascular disease and neurodegenerative disorders [22]. Further research could also explore the efficacy of ET-traps in these other disease areas.

\section{Author contribution}

AJ and VM participated in the research design. AJ, VM and HY conducted the experiments. $\mathrm{AJ}, \mathrm{KH}$, AshokJ and IJ contributed new reagents or analytic tools. VM and AshokJ performed 
the data analysis. AJ, VM, KH, AshokJ, MJ and IJ wrote or contributed to the writing of the manuscript.

\section{Acknowledgements}

We would like to thank DM; Ms. Leela Jain for all her support. We also thank RC for funding this project.

Compliance and ethical standards

Funding

This project was funded by private funding.

\section{Conflict of interest}

None declared. 


\section{References}

1. Nelson, J., et al., The endothelin axis: emerging role in cancer. Nat Rev Cancer, 2003. 3(2): p. 110-6.

2. Struck, J., N.G. Morgenthaler, and A. Bergmann, Proteolytic processing pattern of the endothelin-1 precursor in vivo. Peptides, 2005. 26(12): p. 2482-6.

3. Karet, F. and A. Davenport, Endothelin and the human kidney: a potential target for new drugs. Nephrol Dial Transplant, 1994. 9(5): p. 4.

4. Adachi, M., et al., Functional domains of human endothelin receptor. J Cardiovasc Pharmacol, 1993. 22 Suppl 8: p. S121-4.

5. Takasuka, T., et al., Characterization of endothelin receptors ETA and ETB expressed in COS cells. J Biochem, 1992. 112(3): p. 396-400.

6. Gabbay, E., J. Fraser, and K. McNeil, Review of bosentan in the management of pulmonary arterial hypertension. Vasc Health Risk Manag, 2007. 3(6): p. 887-900.

7. Chaumais, M.C., et al., Clinical pharmacology of endothelin receptor antagonists used in the treatment of pulmonary arterial hypertension. Am J Cardiovasc Drugs, 2015. 15(1): p. 13-26.

8. Hiley, C.R., et al., BQ-123, cyclo(-D-Trp-D-Asp-Pro-D-Val-Leu), is a non-competitive antagonist of the actions of endothelin-1 in SK-N-MC human neuroblastoma cells. Biochem Biophys Res Commun, 1992. 184(1): p. 504-10.

9. Huovinen, T., et al., Two SCFv antibody libraries derived from identical VL-VH framework with different binding site designs display distinct binding profiles. Protein Eng Des Sel, 2013. 26(10): p. 683-93.

10. Patel, S., et al., Selection of a high-affinity WW domain against the extracellular region of VEGF receptor isoform-2 from a combinatorial library using CIS display. Protein Eng Des Sel, 2013. 26(4): p. 307-15.

11. George, R.A. and J. Heringa, An analysis of protein domain linkers: their classification and role in protein folding. Protein Eng, 2002. 15(11): p. 871-9.

12. Aurora, R., et al., Local interactions in protein folding: lessons from the alpha-helix. J Biol Chem, 1997. 272(3): p. 1413-6.

13. Orry, A.J. and B.A. Wallace, Modeling and docking the endothelin G-protein-coupled receptor. Biophys J, 2000. 79(6): p. 3083-94.

14. Dinarello, C.A., Setting the cytokine trap for autoimmunity. Nat Med, 2003. 9(1): p. 20-2.

15. Brazil, M., Setting a trap for cytokines. Nat Rev Drug Discov, 2003. 2(2): p. 90-90.

16. Beck, A. and J.M. Reichert, Therapeutic Fc-fusion proteins and peptides as successful alternatives to antibodies. MAbs, 2011. 3(5): p. 415-6.

17. Schneider, M.P., E.I. Boesen, and D.M. Pollock, Contrasting actions of endothelin ET(A) and $E T(B)$ receptors in cardiovascular disease. Annu Rev Pharmacol Toxicol, 2007. 47: p. 731-59.

18. Fiore, G., et al., Endothelin-1 triggers placental oxidative stress pathways: putative role in preeclampsia. J Clin Endocrinol Metab, 2005. 90(7): p. 4205-10.

19. George, E.M. and J.P. Granger, Endothelin: key mediator of hypertension in preeclampsia. Am J Hypertens, 2011. 24(9): p. 964-9.

20. Tam Tam, K.B., et al., Endothelin type A receptor antagonist attenuates placental ischemiainduced hypertension and uterine vascular resistance. Am J Obstet Gynecol, 2011. 204(4): p. 330 e1-4.

21. Jain, A., et al., Endothelin-1 induces endoplasmic reticulum stress by activating the PLC-IP(3) pathway: implications for placental pathophysiology in preeclampsia. Am J Pathol, 2012. 180(6): p. 2309-20.

22. Jain, A., Endothelin-1-induced endoplasmic reticulum stress in disease. J Pharmacol Exp Ther, 2013. 346(2): p. 163-72. 


\section{Figure legends}

Figure 1. Phage experiments strategy using biotinylated endothelin-1 and endothelin-1 traps (ETtr).

Figure 2. Phage immunoassay for the detection of HA-epitope, done for ETtr1-5. Negative control was a phage stock displaying an unrelated protein (SpyCatcher). The assay was repeated 3 times with replicate phage stocks with similar results.

Figure 3. Phage immunoassay for binding of ETtr1-5 to biotinylated endothelin-1. The negative control was a phage stock displaying an unrelated protein (SpyCatcher). Data represent the mean of three independent experiments with standard deviation. Unspecific binding was analysed in parallel by incubating the phage on streptavidin beads without any coated antigen. Statistical analysis was performed by analysis of variance (Fisher's Protected Least Significant Difference test), with $p<0.001$ being considered significant.

The table below the figure illustrates what LBDs of the ETA receptor constitute (marked with an ' $x$ ') each construct.

Figure $4(A$ and $B)$. SDS-PAGE blots of expressed (A) ETtr1 and (B) ETtr2 constructs. SDS-PAGE analysis of (A) FCETtr1 and (B) Fc-ETtr2 confirmed that both constructs were expressed; identified as per their respective molecular weights (93.7kDa for ETtr1 and 88.9kDA for ETtr2). Both constructs were expressed with a purity $>98 \%$. NR=non-reduced and R=reduced. Non-reduced forms of both ETtr1 and ETtr2 were expressed sufficiently to isolate $5 \mathrm{mg}$ of each at a concentration of $1 \mathrm{mg} / \mathrm{ml}$.

Figure 5. Sensograms of (A) Fc-ETtr1 and (B) Fc-ETtr2 binding to biotinylated ET-1. Representative plots shown for each of the two constructs. Neither Fc-ETtr1 nor Fc-ETtr2 in the current dimer form exhibits any binding to biotinylated ET-1 $(n=3)$.

Figure 6. Sensograms of (A) Fc-ETtr1 and (B) Fc-ETtr2, binding to biotinylated ET-1. Representative plots shown for each of the two constructs. Both constructs are in monomeric form after a mild pre-treatment with $10 \mathrm{mM}$ DTT. ETtr1 gave a mean $K_{D}$ of $21.2 \mathrm{nM}(n=6)$ and ETtr2 gave a mean $K_{D}$ of $77.3 \mathrm{nM}(\mathrm{n}=3)$.

Figure 7. Possible formats of Fc-fusion constructs, with the black circle being the fusion protein. 


\section{Figures}

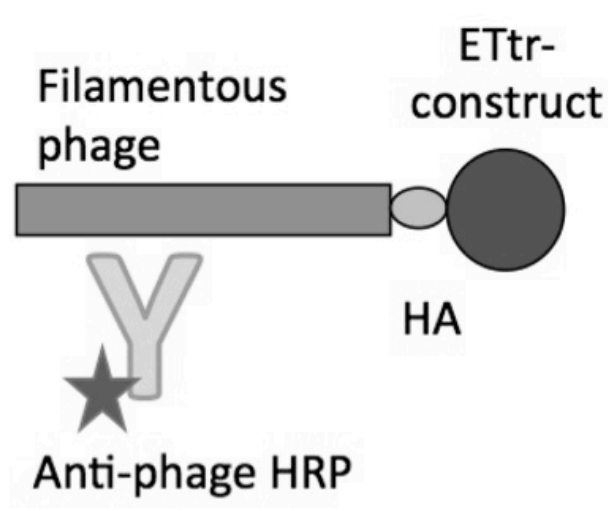

Bio-endothelin-1

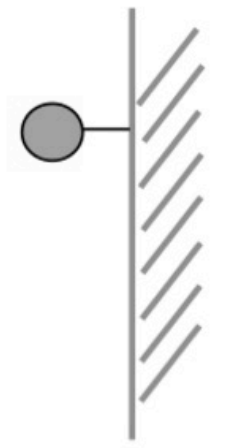

Figure 1.

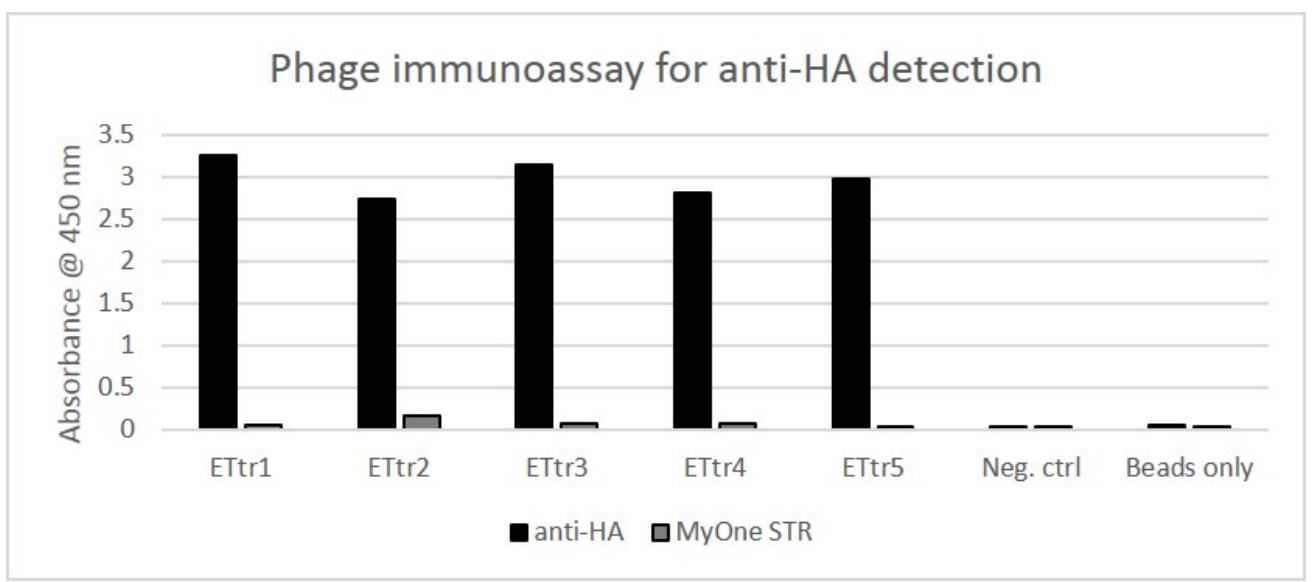

Figure 2. 
Creating a soluble binder to ET-1

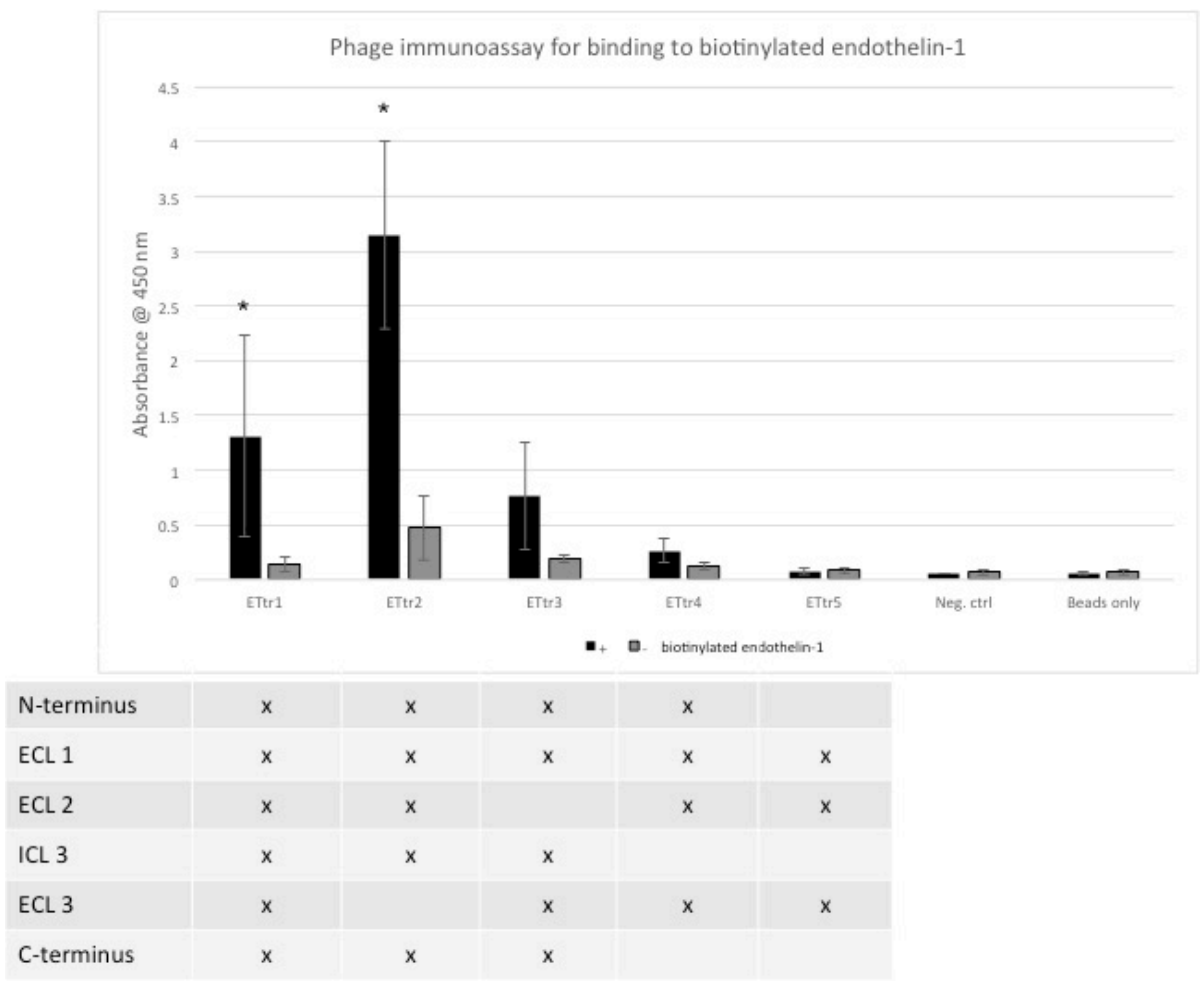

Figure 3.

\section{(A)}

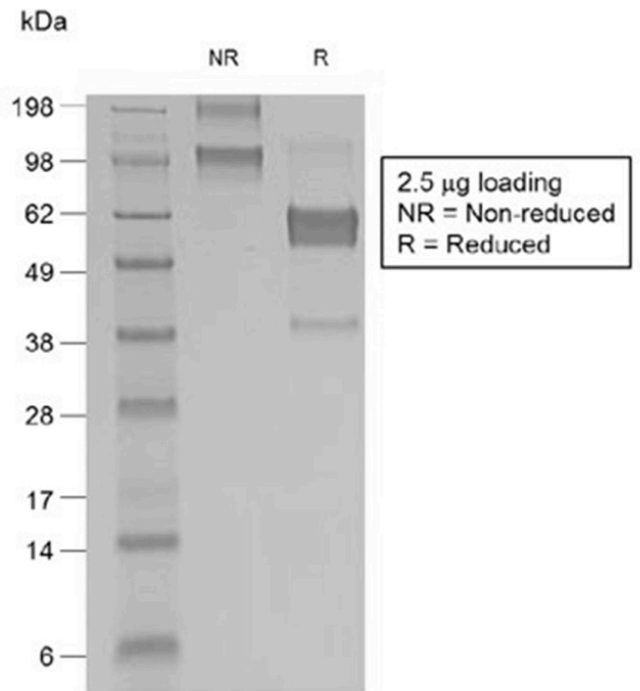

(B)

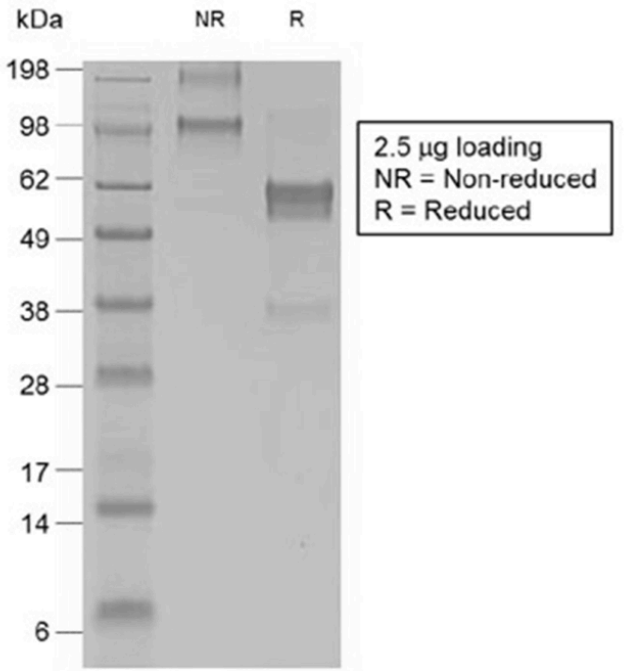

Figure 4 ( $A$ and $B)$ 


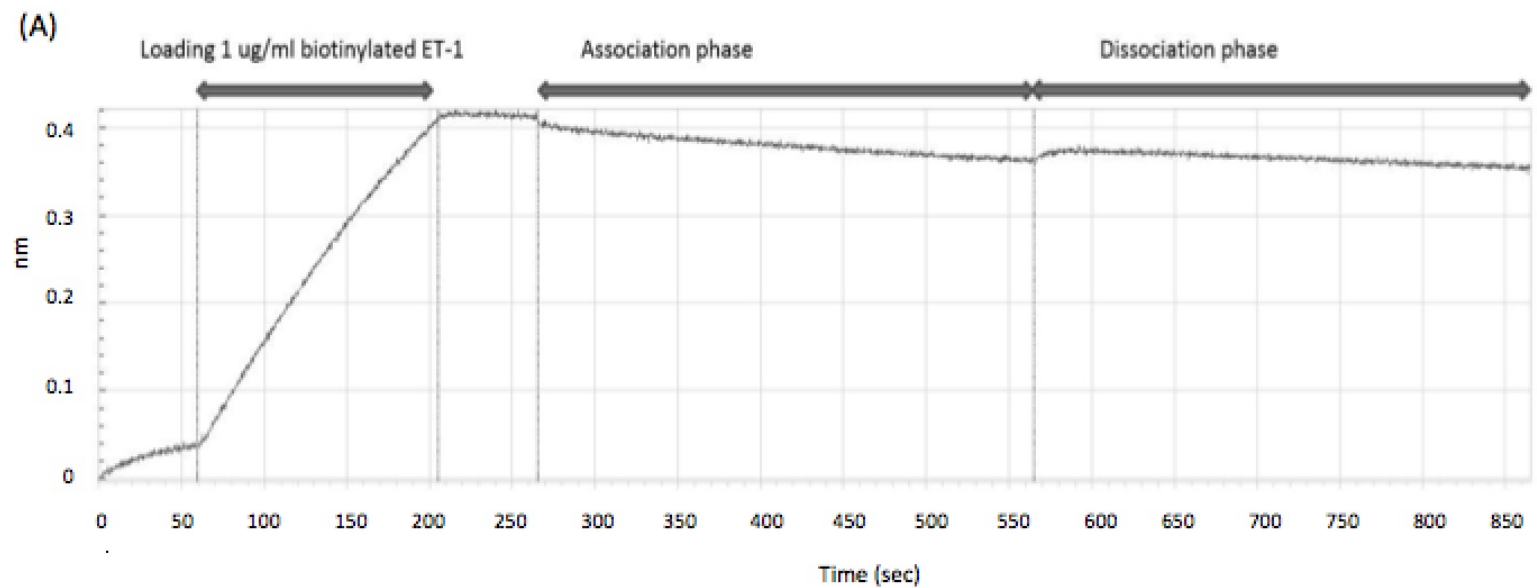

(B)

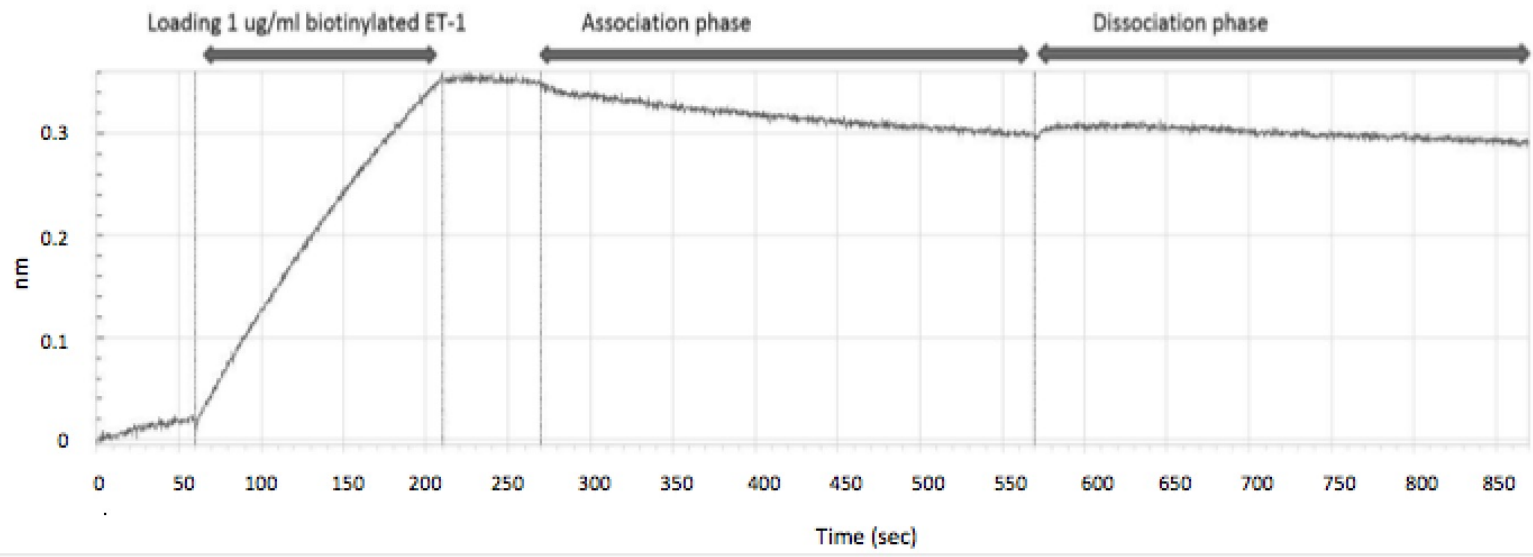

Figure 5 ( $A$ and $B$ ) 
(A)

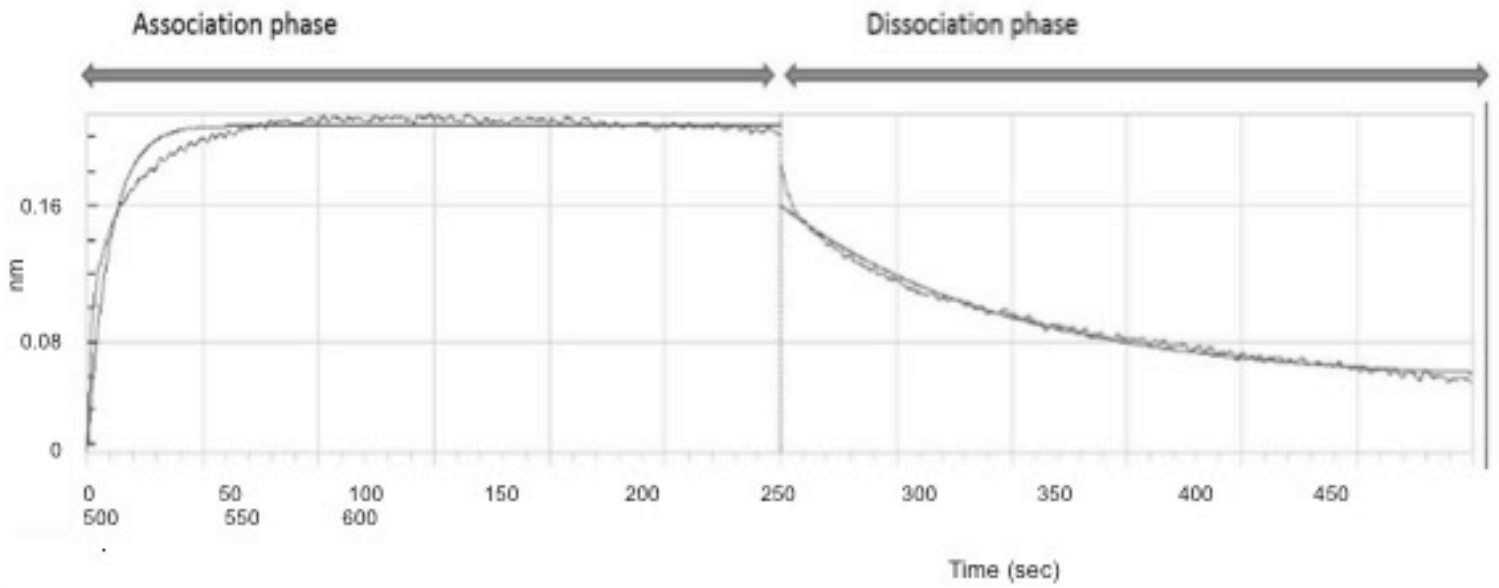

(B)

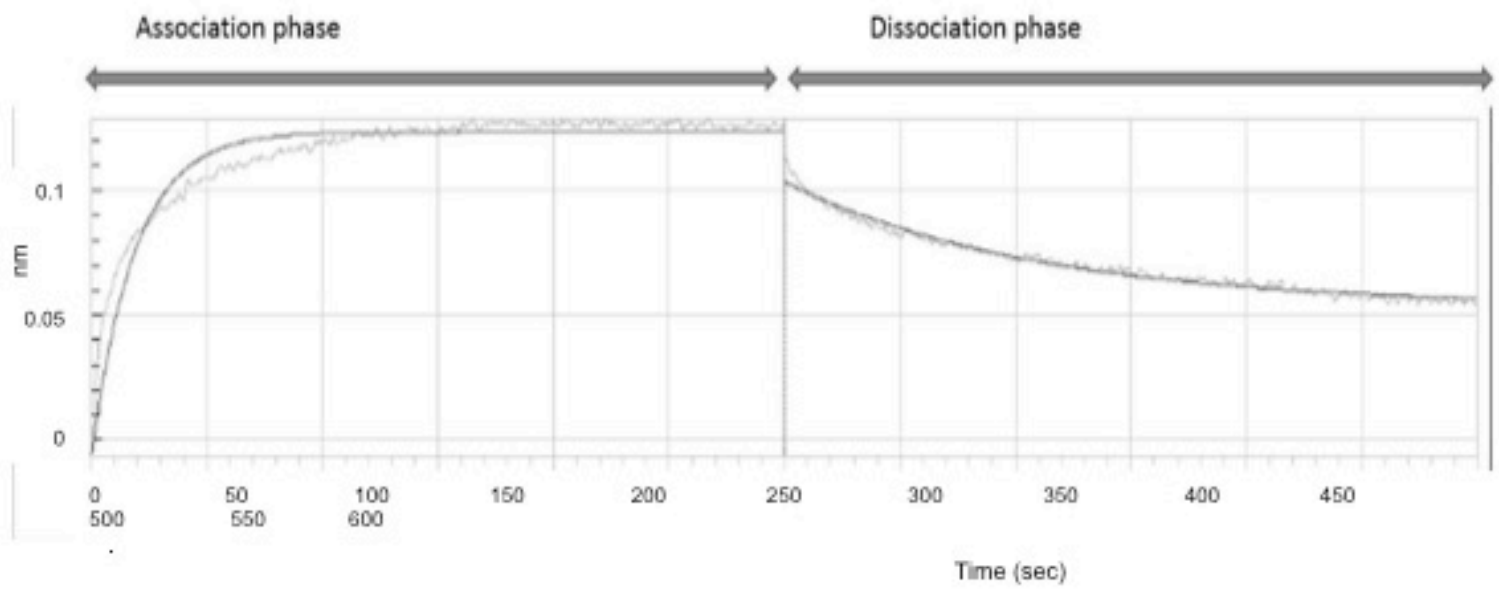

Figure 6 ( $A$ and $B$ ) 
Creating a soluble binder to ET-1
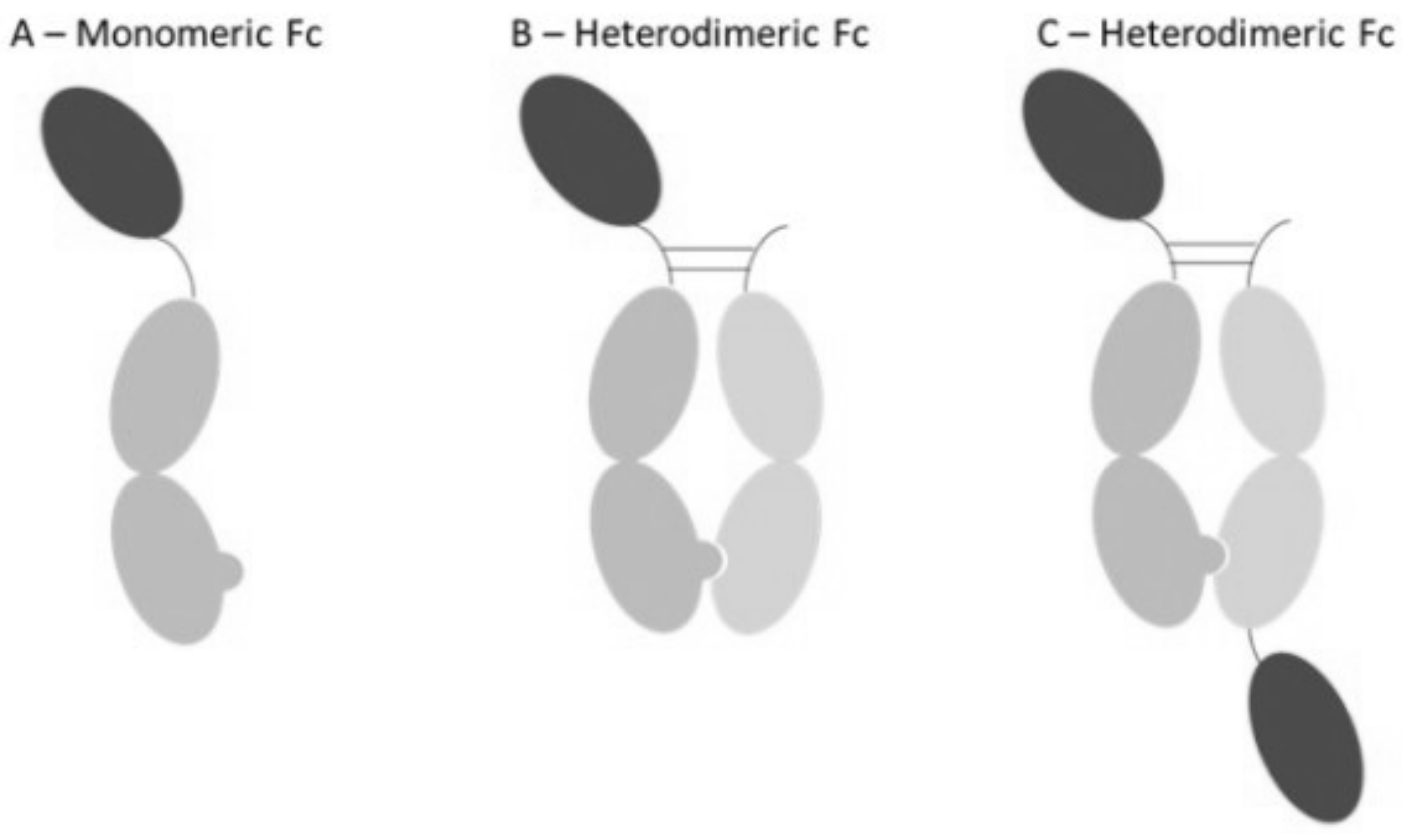

Figure 7. 